\title{
The effect of glycogen reduction on cardiorespiratory and metabolic responses during downhill running
}

James Peter Gavin 1

Stephen David Myers 1

Mark Elisabeth Theodorus Willems 1,*

Phone $+44(0) 1243816468$

Emailm.willems@chi.ac.uk

Department of Sport and Exercise Sciences, University of Chichester, College Lane, Chichester, PO19 6PE UK

\section{Abstract \\ Purpose}

Exercise-induced muscle damage and lowered glycogen are common during heavy training periods, and may prolong recovery. We examined the effects of lowered glycogen on cardiorespiratory, metabolic and perceptual responses to downhill running.

\section{Methods}

Twelve men performed two downhill runs $(-12 \%$ gradient, $12.1 \pm 1.1 \mathrm{~km} \mathrm{~h}^{-1}$ ) separated by 6 weeks, under normal (NORM) and reduced glycogen (RED) conditions in a crossover design. For RED, participants performed exhaustive cycling at $60 \% \dot{V} \mathrm{O}_{2 \max }$ power $(95 \pm 13 \mathrm{~min})$ in the evening, and the next morning completed a downhill run comprising of five stages of 8 min running, with $2 \mathrm{~min}$ recovery ( $1 \%$ gradient, $8 \mathrm{~km} \mathrm{~h}^{-1}$ ) between each stage. Expired gas, heart rate, rating of perceived exertion (RPE) and blood lactate (bLa) and glucose were measured for each stage. 


\section{Results}

Blood glucose $(P<0.05)$ and respiratory exchange ratio $(P<0.01)$ were lower in RED, than NORM, throughout the downhill run. RED demonstrated higher bLa until stage Four $(P<0.05)$, and RPE for stages Two and Five $(P<0.05)$.Ventilatory equivalent of carbon dioxide output $\left(\dot{V}_{E} / \dot{V} \mathrm{CO}_{2}\right)$ was higher for stages One $(P<0.01)$, Two and Five $(P<0.05)$, and oxygen uptake $\left(\dot{V}_{\mathrm{E}} / \dot{V} \mathrm{O}_{2}\right)$ was lower for stages Three and Four $(P<0.05)$ for RED.

\section{Conclusions}

Downhill running with reduced glycogen, elevated fat oxidation and bLa response, and, in part, increased effort perception. The alterations in $\dot{V}_{\mathrm{E}} / \dot{V} \mathrm{O}_{2}$ and bLa may suggest that carbon dioxide removal was somewhat impaired.

\section{Keywords}

Muscle damage

Glycogen

Exercise metabolism

Eccentric exercise

Effort perception

Respiration

Abbreviations

bLa Blood lactate

DOMS Delayed onset muscle soreness

HR Heart rate

NORM Normal glycogen

RED Reduced glycogen

RER Respiratory exchange ratio

RPE Rating of perceived exertion

$\dot{V} \mathrm{CO}_{2} \quad$ Carbon dioxide output

$\dot{V}_{\mathrm{E}} \quad$ Minute ventilation 
$\dot{V}_{\mathrm{E}} / \dot{V} \mathrm{CO}_{2}$ Ventilatory equivalent of carbon dioxide

$\dot{V}_{\mathrm{E}} / \dot{V} \mathrm{O}_{2} \quad$ Ventilatory equivalent of oxygen uptake

$\dot{V} \mathrm{O}_{2} \quad$ Oxygen uptake

$\dot{V} \mathrm{O}_{2 \max } \quad$ Maximal oxygen uptake

Communicated by Michael Lindinger.

\section{Introduction}

Prolonged, intense or unaccustomed exercise is known to induce muscle damage, leading to force loss, muscle soreness (Clarkson and Sayers 1999) and impaired glucose metabolism (Asp et al. 1995; Krishnan et al. 2003 ). After prolonged eccentric exercise, glycogen stores remain depleted up to 10 days (O'Reilly et al. 1987). It appears that eccentricbiased exercise not only disrupts muscle glycogen uptake and resynthesis, but does so in a delayed manner (Widrick et al. 1992). Altered glucose metabolism is known to result from exercise-induced insulin resistance, as reflected by decreased fat oxidation and preserved carbohydrate oxidation at rest in young men (Krishnan et al. 2003).

Earlier studies examining glycogen depletion had participants perform submaximal, exhaustive cycling (between 85 and $135 \mathrm{~min}$ ) in the evening, followed by an overnight fast (Hughes et al. 1982; Segal and Brooks 1979). During moderate and heavy exercise the next morning, attenuated responses were shown for blood lactate (bLa), carbon dioxide output ( $\dot{V} \mathrm{CO}_{2}$ ) (Segal and Brooks 1979), respiratory exchange ratio (RER) and the ventilatory equivalent of oxygen uptake $\left(\dot{V}_{\mathrm{E}} / \dot{V} \mathrm{O}_{2}\right)$ (Hughes et al. 1982). Glycogen depletion protocols have also been used to manipulate muscle fibre recruitment patterns prior to moderate-intensity exercise (Krustrup et al. 2004). Selectively depleting slow-twitch fibres resulted in enhanced fast-twitch fibre recruitment and increase oxygen uptake $\left(\dot{V} \mathrm{O}_{2}\right)$ during subsequent $50 \%$ maximal oxygen uptake $\left(\dot{V} \mathrm{O}_{2 \max }\right)$ cycling. However, bLa accumulation was unchanged. It is also plausible that reducing muscle glycogen may increase fat oxidation in slow-twitch fibres for subsequent exercise. For example, lowering slow-twitch fibre glycogen content prior to heavy-intensity exercise has been shown to increase $\dot{V} \mathrm{O}_{2}$ and fat oxidation, without altering $\dot{V} \mathrm{O}_{2}$ kinetics or fibre recruitment 
patterns (Bouckaert et al. 2004). These authors reported respiratory and metabolic measures for concentric-biased exercise with lowered muscle glycogen, yet none have examined responses during eccentric-biased exercise with lowered glycogen.

Exercise-induced muscle damage and lowered glycogen are common during intense training periods. Muscle damage from downhill running has been shown to increase $\dot{V} \mathrm{O}_{2}$, perceived exertion (Baumann et al. 2014), bLa and RER responses of young men undergoing subsequent level running (Hughes et al. 1982). Downhill running involves the knee extensors undergoing repetitive, intense lengthening contractions to decelerate the centre of mass, through a large range of motion (Eston et al. 1995 ). In this regard, downhill running represents a valid exercise mode to induce muscle damage that has particular application for endurance athletes, such as marathon and cross-country runners who experience trauma from surface impact and the changing terrain. As such, it is important to understand how a bout of exercise-inducing muscle damage is influenced by pre-exercise glycogen stores. Glycogen depletion appears to attenuate respiratory and metabolic responses to concentric exercise (Hughes et al. 1982; Segal and Brooks 1979). However, due to different physiological demands, the same cannot be expected for eccentric aerobic exercise. Downhill running incurs lower cardiovascular (Meyer et al. 2002 AQ2

) and energetic cost, in comparison to concentric exercise (Minetti et al. 2002), but does impair glucose metabolism after the event. Performing eccentric-biased, aerobic exercise with reduced glycogen, may promote our understanding of the role that substrate availability plays in the degenerative and regenerative processes of healthy, functioning muscle. Based upon the abovementioned findings, our working hypothesis states that pre-exercise glycogen reduction would increase bLa and cardiorespiratory responses for, and incurred damage from, subsequent muscle-damaging exercise. Therefore, the study aim was to investigate the effects of reduced muscle glycogen on the cardiorespiratory, metabolic and perceptual responses to muscle-damaging, downhill running in young, healthy men.

\section{Method}




\section{Participants}

Twelve healthy men (Table 1) provided written informed consent for study participation. Sample size estimation was based on maximal isometric force loss $(-15 \%, \alpha$ level $=0.05$, power $=0.80)($ Faul et al. 2007 $)$. Volunteers were recreationally active and had no history of structured resistance and/or regular running training. As confirmed by a medical history questionnaire, all were free from joint and musculoskeletal injury. The research protocol was approved by the University of Chichester Research Ethics Committee and conducted in accordance with the Helsinki Declaration.

\section{Table 1}

Participant characteristics $(n=12)$

\begin{tabular}{|c|c|}
\hline & Mean \pm SD \\
\hline Age (years) & $23 \pm 4$ \\
\hline Height $(\mathrm{cm})$ & $179 \pm 5$ \\
\hline Body mass (kg) & $76.6 \pm 9.8$ \\
\hline BMI $\left(\mathrm{kg} \mathrm{m}^{-2}\right)$ & $23.9 \pm 2.7$ \\
\hline Body fat (\%) & $14.4 \pm 3.8$ \\
\hline$\dot{V} \mathrm{O}_{2 \max }\left(\mathrm{L} \mathrm{min}^{-1}\right)$ & $4.1 \pm 1.0$ \\
\hline$\dot{V} \mathrm{O}_{2 \max }\left(\mathrm{mL} \mathrm{kg}^{-1} \min ^{-1}\right)$ & $54 \pm 9$ \\
\hline Maximal workload (W) & $314 \pm 57$ \\
\hline Glycogen reduction cycling workload (W) & $181 \pm 40$ \\
\hline Downhill running speed $\left(\mathrm{km} \mathrm{h}^{-1}\right)$ & $12.1 \pm 1.1$ \\
\hline Training time per week (h) & $5.8 \pm 3.3$ \\
\hline Training sessions per week & $4 \pm 2$ \\
\hline
\end{tabular}

\section{Experimental design}

Cardiorespiratory responses were measured during two, downhill treadmill runs under (1) normal glycogen (NORM), and (2) reduced glycogen (RED) 
conditions. A randomised cross-over design was used, with each downhill run separated by at least 6 weeks. Blind selection was used to randomly allocate run order (NORM first, $n=5$; RED first, $n=7$ ), and only the first run was preceded by familiarisations. A repeat bout of the downhill running protocol has been shown not to affect knee extensor strength in a similar cohort 5 weeks later (Eston et al. 2000). Similarly, we found no order effect between downhill runs for maximal force loss $(P=0.12$, $d=0.11$ ). The NORM involved a resting evening session, followed by a morning run; RED involved an exhaustive, cycling exercise evening session, followed by a morning downhill run. Instruction was given to arrive hydrated, having abstained from heavy physical activity $48 \mathrm{~h}$ prior to the evening testing session. Aside from water ad libitum, food was not permitted until after the downhill run the next morning. Dietary manipulation required participants to arrive for the evening visits $3 \mathrm{~h}$ after their final meal (similar blood glucose, $P>0.05$ ), having not consumed caffeine in the preceding $12 \mathrm{~h}$. Habitual diet was maintained and selfrecorded from $48 \mathrm{~h}$ prior to the first experimental condition, up to $48 \mathrm{~h}$ after the downhill run. Food records were checked by the investigator upon each visit and prescribed for the subsequent condition, as was physical activity, which was requested to remain low, according to the International Physical Activity Questionnaire (Craig et al. 2003 ).

\section{Baseline measures}

Familiarisation one involved anthropometric and resting measurements, followed by a maximal, incremental cycling test to prescribe intensity for glycogen reduction cycling. The cycling test was performed on an electronically controlled ergometer (Excalibur Sport 925900, Lode, Groningen, The Netherlands), with participants maintaining a $\sim 75 \mathrm{rpm}$ cadence at $50 \mathrm{~W}$ for $3 \mathrm{~min}$, and thereafter $10 \mathrm{~W}$ increments every $20 \mathrm{~s}$ until volitional exhaustion (Osborne and Schneider 2006). A portable metabolic cart (Cosmed $\mathrm{K} 4 \mathrm{~b}^{2}$, Rome, Italy) sampled expired gas breath by breath to

determine $\dot{V} \mathrm{O}_{2 \mathrm{max}}$, which was used to prescribe individual workload for glycogen reduction cycling.

Familiarisation two occurred $\sim 48 \mathrm{~h}$ after the first, and $\sim 48 \mathrm{~h}$ before the initial, evening experimental condition. A submaximal, incremental running test was performed to establish downhill running speed. Heart rate (HR), bLa and glucose were sampled after a 5 min seated rest, then 
participants warmed up for 5 min at $8 \mathrm{~km} \mathrm{~h}^{-1}$ (1\% gradient) on a precalibrated motorised treadmill (Pulsar, $\mathrm{h} / \mathrm{p} / \operatorname{cosmos}$ Sports and Medical $\mathrm{GmbH}$, Germany). The test started at the warm-up speed, which was increased by $1 \mathrm{~km} \mathrm{~h}^{-1}$ every 4 min, until (1) eight stages, or (2) volitional exhaustion was reached. Expired gas was collected in the final $60 \mathrm{~s}$ of each stage with 200 L Douglas bags (Cranlea and Co., Birmingham, UK); HR (Polar Electro Oy, Kempele, Finland), rating of perceived exertion (RPE) (Borg 1998), and a fingertip capillary blood sample were measured in the final $30 \mathrm{~s}$. Capillary blood $(25 \mu \mathrm{L})$ was sampled from the index finger into EDTA-coated microvettes (SarstedtAktiengesellsehaftSarstedt

Aktiengesellschaft and Co., Nümbrecht, Germany). Blood samples were analysed immediately for glucose and bLa concentrations using a precalibrated analyser (2300 STAT Plus ${ }^{\mathrm{TM}}$ analyser, YSI Life Sciences, Yellow Springs, USA), and then software (Newell et al. 2007) was used to estimate individual lactate threshold to prescribe downhill running speed.

\section{Glycogen reduction cycling protocol}

Participants reported to the laboratory in the evening (between 1905 and 1950 hours), $3 \mathrm{~h}$ after a light meal, to perform a preliminary cycling bout to lower muscle glycogen. After a $10 \mathrm{~min}$ warm up ( $75 \mathrm{rpm}$ at $50 \%$ $\dot{V} \mathrm{O}_{2 \max }$ ), participants cycled at $60 \% \dot{V} \mathrm{O}_{2 \max }$ until volitional exhaustion (mean time, $95 \pm 13 \mathrm{~min}$ ), as confirmed by an inability to maintain a $50 \mathrm{rpm}$ cadence. Expired gas, HR, RPE and fingertip blood were measured every 20 min (Table 2 ). The protocol was modified from previous studies (Osborne and Schneider 2006; Thomson et al. 1979), having used biopsy to confirm muscle fibre glycogen depletion (slow twitch, $95 \%$; fast twitch, $70 \%$ ) (Thomson et al. 1979). For NORM, participants reported to the laboratory in the evening, provided resting measurements after a $5 \mathrm{~min}$ rest, and then remained seated in a dimly-lit room for a period of $2 \mathrm{~h}$. Arrival times and dietary instruction were matched between the randomised conditions.

\section{Table 2}

Mean physiological responses to glycogen reduction cycling

\begin{tabular}{|l|l|l|l|l|l|}
\hline & $\begin{array}{l}\mathbf{2 0} \text { min } \\
(\boldsymbol{n}=\mathbf{1 2})\end{array}$ & $\begin{array}{l}\mathbf{4 0} \mathbf{\text { min }} \\
(\boldsymbol{n}=\mathbf{1 2})\end{array}$ & $\begin{array}{l}\mathbf{6 0} \mathbf{\text { min }} \\
(\boldsymbol{n}=\mathbf{1 2})\end{array}$ & $\begin{array}{l}\mathbf{8 0} \mathbf{\text { in }} \\
(\boldsymbol{n}=\mathbf{1 2})\end{array}$ & $\begin{array}{l}\mathbf{1 0 0} \mathbf{\text { min }} \\
(\boldsymbol{n}=\mathbf{7})\end{array}$ \\
\hline Workload $(W)$ & $181 \pm 32$ & $174 \pm 29$ & $165 \pm 28$ & $163 \pm 28$ & $170 \pm 34$ \\
\hline Cadence & & & & & \\
\hline
\end{tabular}




\begin{tabular}{|c|c|c|c|c|c|}
\hline (rpm) & $74 \pm 2$ & $73 \pm 2$ & $73 \pm 2$ & $73 \pm 2$ & $79 \pm 1$ \\
\hline$\dot{V}_{\mathrm{E}}\left(\mathrm{L} \min ^{-1}\right)$ & $77.9 \pm 14.2$ & $78.3 \pm 16.0$ & $76.6 \pm 16.8$ & $80.8 \pm 17.4$ & $73.5 \pm 14$ \\
\hline$\dot{V}_{1} \mathrm{O}_{2}(\mathrm{~L} \min$ & $2.56 \pm 0.38$ & $2.59 \pm 0.49$ & $2.57 \pm 0.49$ & $2.56 \pm 0.76$ & $2.66 \pm 0$. \\
\hline$\dot{V}_{-1} \mathrm{CO}_{2}(\mathrm{~L} \min$ & $2.44 \pm 0.21$ & $2.4 \pm 0.28$ & $2.32 \pm 0.24$ & $2.36 \pm 0.27$ & $2.21 \pm 0.2$ \\
\hline $\begin{array}{l}\dot{V} \mathrm{O}_{2} \\
\left(\mathrm{~mL}^{2} \mathrm{~kg} \mathrm{~min}^{-1}\right)\end{array}$ & $33.7 \pm 6.8$ & $34.1 \pm 8.2$ & $33.8 \pm 8.4$ & $33.8 \pm 12.1$ & $35.8 \pm 11$ \\
\hline $\mathrm{FeO}_{2}(\%)$ & $16.8 \pm 1.0$ & $16.8 \pm 1.0$ & $16.8 \pm 1.1$ & $17.0 \pm 1.2$ & $16.6 \pm 1.4$ \\
\hline $\mathrm{FeCO}_{2}(\%)$ & $3.9 \pm 0.6$ & $3.8 \pm 0.6$ & $3.8 \pm 0.6$ & $3.7 \pm 0.5$ & $3.7 \pm 0.5$ \\
\hline $\begin{array}{l}\dot{V}_{\mathrm{E}} / \dot{\mathrm{V}}^{\mathrm{O}} \mathrm{O}_{2} \\
\left.\mathrm{~min}^{-1}\right)\end{array}$ & $31.1 \pm 8.0$ & $31.3 \pm 9.1$ & $30.8 \pm 9.0$ & $34.3 \pm 14.5$ & $29.7 \pm 10$ \\
\hline $\begin{array}{l}\dot{V}_{\mathrm{E}} / \dot{\vec{V}} \mathrm{CO}_{2} \\
\left(\mathrm{Lin}^{-1}\right)\end{array}$ & $31.8 \pm 4.4$ & $32.4 \pm 4.8$ & $32.8 \pm 5.4$ & $33.9 \pm 4.3$ & $33.3 \pm 4$. \\
\hline RER & $0.95 \pm 0.12$ & $0.94 \pm 0.15$ & $0.92 \pm 0.15$ & $0.91 \pm 0.16$ & $0.88 \pm 0.2$ \\
\hline$\left.{ }_{-1}^{\mathrm{bLa}}\right)(\mathrm{mmol} \mathrm{L}$ & $3.77 \pm 1.56$ & $2.62 \pm 0.83$ & $2.67 \pm 0.63$ & $2.84 \pm 1.08$ & $2.26 \pm 1 . \xi$ \\
\hline $\begin{array}{l}\text { Glucose } \\
\left(\mathrm{mmol} \mathrm{L}^{-1}\right)\end{array}$ & $3.83 \pm 0.40$ & $3.37 \pm 0.29$ & $3.39 \pm 0.39$ & $3.18 \pm 0.49$ & $2.96 \pm 0 .\llcorner$ \\
\hline $\operatorname{HR}\left(\mathrm{b} \min ^{-1}\right)$ & $162 \pm 9$ & $162 \pm 9$ & $162 \pm 6$ & $162 \pm 10$ & $157 \pm 15$ \\
\hline RPE & $13.4 \pm 1.5$ & $15.5 \pm 1.2$ & $16.6 \pm 1.5$ & $18.1 \pm 1.1$ & $18.4 \pm 0.5$ \\
\hline
\end{tabular}

Values are mean $\pm \mathrm{SD}$

$\dot{V}_{\mathrm{E}}$ minute ventilation, $\dot{V} \mathrm{O}_{2}$ oxygen uptake, $\dot{V} \mathrm{CO}_{2}$ carbon dioxide production, $\mathrm{F} \epsilon$ of expired oxygen, $\mathrm{FeCO}_{2}$ fraction of expired carbon dioxide, $\dot{V}_{E} / \dot{V} \mathrm{O}_{2}$ ventilator: of oxygen uptake, $\dot{V}_{\mathrm{E}} / \dot{V} \mathrm{CO}_{2}$ ventilatory equivalent of carbon dioxide production, respiratory exchange ratio, $b L a$ blood lactate, $H R$ heart rate, $R P E$ rating of perceiv $n$ indicates number of subjects at timepoint of the glycogen reduction cycling proti

\section{Downhill running}

Participants attended the laboratory in the morning (between 0650 and 0715 hours), to perform downhill running ( $-12 \%$ gradient) at individual lactate threshold speed $\left(12.1 \pm 1.1 \mathrm{~km} \mathrm{~h}^{-1}\right)$. After a 5 min warm up $(1 \%$ gradient at $8 \mathrm{~km} \mathrm{~h}^{-1}$ ), participants performed five, 8 min stages of downhill running, separated by 2 min recovery periods (1 \% gradient at 
$\left.8 \mathrm{~km} \mathrm{~h}^{-1}\right)$. Previously used with young individuals, at a fixed running speed $\left(11.3 \mathrm{~km} \mathrm{~h}^{-1}\right)$, the protocol was effective in inducing knee extensor soreness and maximal force loss up to $72 \mathrm{~h}$ later (Eston et al. 2000). Expired gas was sampled in the final $60 \mathrm{~s}$ of each stage, and HR, RPE and fingertip blood in the final $30 \mathrm{~s}$. Between conditions, the same footwear and treadmill were used in a temperature controlled laboratory $\left(19-21^{\circ} \mathrm{C}\right)$.

Knee extensor soreness and maximal force were measured on a custombuilt, strength-testing chair with hip and knee angle at $1.57 \mathrm{rad}$, before and after downhill running (at 0 and $48 \mathrm{~h}$ ). Soreness was assessed using a visual analogue scale $(0=$ not at all sore; $10=$ extremely sore $)$, with the muscle-belly palpated until enough pressure was exerted to blanch the fingernail. Force was measured during three separate, 3-5 s maximal isometric contractions ( 2 min rests), with verbal encouragement and forcetime feedback provided by the computer monitor.

\section{Statistical analysis}

A paired sample $t$ test was used to examine whether there was an order effect by analysis of maximal force loss and soreness at $48 \mathrm{~h}$ for bout 1 and 2. A two-way repeated measures ANOVA was used to compare physiological responses during each stage of downhill running, and maximal force loss, between NORM and RED conditions. Differences were located using pre-planned $t$ tests with a Bonferroni correction used to control for multiple comparisons. Where assumptions of sphericity were violated, a Greenhouse-Geisser correction was applied. Friedman tests were used on non-parametric: muscle soreness, RPE, $\dot{V}_{\mathrm{E}} / \dot{V}^{\mathrm{O}_{2}}$ and $\dot{V}_{\mathrm{E}} /$ $\dot{V} \mathrm{CO}_{2}$ data, with Wilcoxon signed-rank tests locating individual stage differences. Effect sizes (Cohen's $d$ ) were calculated for all significant post hoc tests of parameters recorded during downhill running. These were interpreted as small (0.2-0.3), moderate (0.31-0.79) and large $(\geq 0.8)$ effect size (Thomas et al. 1991). Data are presented as mean $\pm \mathrm{SD}$, with an alpha level of $<0.05$.

\section{Results}

\section{Markers of muscle damage}

Our experimental design did not show an order effect as post-exercise, the force loss at $48 \mathrm{~h}$ (bout 1: $-19 \pm 1 \%$, bout $2:-15 \pm 18 \%, P=0.43$ ), and 
muscle soreness at $48 \mathrm{~h}$ (bout 1: $3.6 \pm 1.6$, bout 2: $4.5 \pm 2.0, P=0.12$ ) were similar. Maximal isometric force loss from downhill running was similar between conditions $(P>0.05)$, immediately after (NORM, $-24 \pm 15 \%$; RED, $-27 \pm 10 \%$ ) but higher at $48 \mathrm{~h}$ for RED (NORM, $-12 \pm 14 \%$; RED, $-23 \pm 14 \%, P<0.05)$. Muscle soreness showed a significant effect; soreness was higher for RED (3.9 \pm 1.8$)$, than NORM $(3.0 \pm 1.2 ; P<0.05)$ immediately after, but similar $48 \mathrm{~h}$ after (NORM, $3.8 \pm 1.2 ;$ RED $, 4.4 \pm 2.3, P=0.37)$.

\section{Blood lactate concentration}

During downhill running, bLa was significantly different between conditions $(P<0.05)$ with no interaction effect. Resting bLa was moderately higher for RED $\left(0.84 \pm 0.23 \mathrm{mmol} \mathrm{L}^{-1}\right)$, than for NORM $\left(0.71 \pm 0.23 \mathrm{mmol} \mathrm{L}^{-1}, P<0.05, d=0.62\right.$; Fig. 1$)$. After stage One, bLa was $2.14 \pm 0.93 \mathrm{mmol} \mathrm{L}^{-1}$ for RED, and $1.59 \pm 0.46 \mathrm{mmol} \mathrm{L}^{-1}$ for NORM $(P<0.05, d=0.75$, moderate effect size $)$. From stages Two to Three, bLa had increased from $2.13 \pm 0.57$ to $2.54 \pm 1.04 \mathrm{mmol} \mathrm{L}^{-1}$ for RED, and from $1.47 \pm 0.55(d=1.18$, large effect size $)$ to $1.61 \pm 0.53 \mathrm{mmol} \mathrm{L}^{-1}$ $(d=1.14$, large effect size) for NORM respectively. Thereafter, mean bLa was similar between RED and NORM (Fig. 1).

Fig. 1

Blood lactate concentrations (bLa) at rest, and during downhill running with normal (NORM) and reduced (RED) glycogen. Values are mean \pm SD. *Significant difference between conditions, $P<0.05$

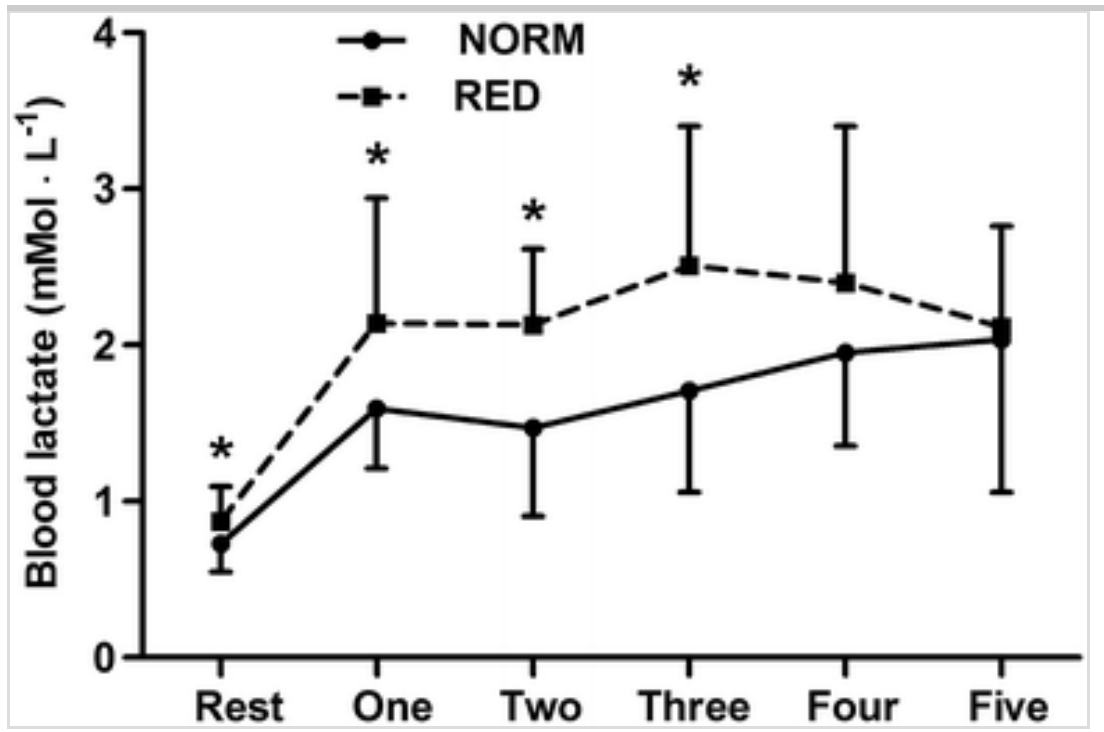


During downhill running, blood glucose concentration showed a significant condition-time interaction effect $(P=0.03)$. Pre-downhill, glucose

(Fig. 2) was lower in RED than in NORM $\left(3.66 \pm 0.44 \mathrm{mmol} \mathrm{L}^{-1}\right.$, NORM, $4.19 \pm 0.64 \mathrm{mmol} \mathrm{L}^{-1} ; P<0.01, d=1.03$, large effect size). Blood glucose remained lower in RED, than in NORM, from stages One $\left(3.63 \pm 0.28 \mathrm{mmol} \mathrm{L}^{-1}\right.$; NORM, $4.03 \pm 0.42 \mathrm{mmol} \mathrm{L}^{-1}, P<0.05, d=1.33$, large effect size $)$ to Five $\left(3.65 \pm 0.53 \mathrm{mmol} \mathrm{L}^{-1}\right.$; NORM, $4.63 \pm 0.56 \mathrm{mmol} \mathrm{L}^{-1}, P<0.01, d=1.80$, large effect size) of downhill running.

Fig. 2

Blood glucose concentrations at rest, and during downhill running with normal $(N O R M)$ and reduced $(R E D)$ glycogen. Values are mean $\pm \mathrm{SD}$. *Significant difference between conditions, $P<0.05$. ${ }^{\#}$ Trend, $P=0.08$

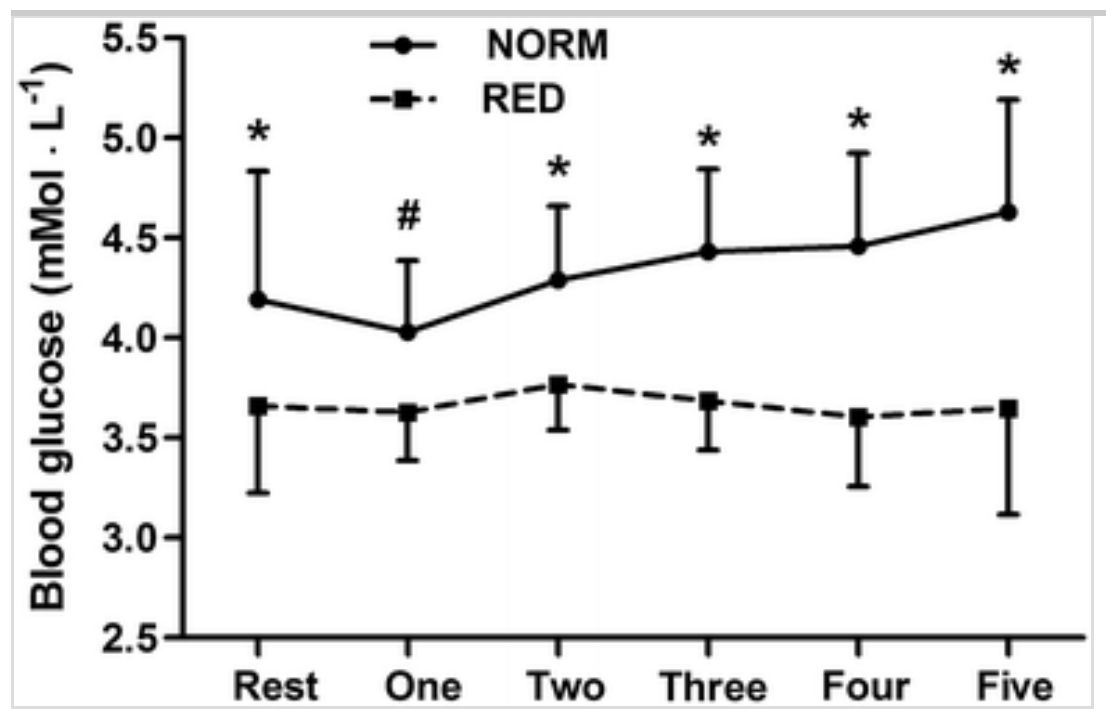

\section{Cardiorespiratory and perceptual responses}

There were no consistent differences between conditions for minute ventilation $\left(\dot{V}_{\mathrm{E}}\right), \dot{V} \mathrm{O}_{2}, \dot{V} \mathrm{CO}_{2}$ or $\mathrm{HR}(P>0.05)$. During running, $\dot{V}_{\mathrm{E}}$ was similar, increasing by $4.8 \mathrm{~L} \mathrm{~min}^{-1}$ for $\operatorname{RED}(d=0.40)$ and $6.2 \mathrm{~L} \mathrm{~min}^{-1}$ for NORM by stage Five $(P<0.01, d=0.57$, moderate effect size; Table 3$)$. Similarly, $\dot{V} \mathrm{O}_{2}$ and $\dot{V} \mathrm{CO}_{2}$ increased by stage Five in both conditions $(P<0.05$; Table 3$)$. Mean $\dot{V}_{\mathrm{E}} / \dot{V} \mathrm{O}_{2}$ was different between conditions $(P<0.05)$, lower for RED, than NORM, at stages Three $(P<0.05$, $d=0.49$, moderate effect size $)$ and Four $(P<0.05, d=0.70$, moderate effect size; Table 3$)$. Mean $\dot{V}_{\mathrm{E}} / \dot{V} \mathrm{CO}_{2}$ was significantly higher for RED, than NORM $(P<0.05)$, during stages One $\left(2.0 \mathrm{~L} \mathrm{~min}^{-1}, P<0.01\right.$, 
$d=0.65$, moderate effect size), Two $\left(1.4 \mathrm{~L} \mathrm{~min}^{-1}, P<0.05, d=0.51\right.$, moderate effect size) and Five $\left(1.0 \mathrm{~L} \mathrm{~min}^{-1}, P<0.05, d=0.30\right.$, small effect size). For RED, HR appeared higher than NORM in stage Two $(P<0.05, d=0.20$, small effect size $)$, and was higher in stage Five, than stage One in both conditions (Table 3 ) with no interaction effect. RPE was significantly $(P<0.001)$ higher in stages Two $(P<0.05, d=0.58$, moderate effect size $)$ and Five $(P<0.05, d=0.36$, moderate effect size $)$ with no interaction effect.

\section{Table 3}

Cardiorespiratory, metabolic and perceptual responses during downhill running in reduced (RED) glycogen conditions

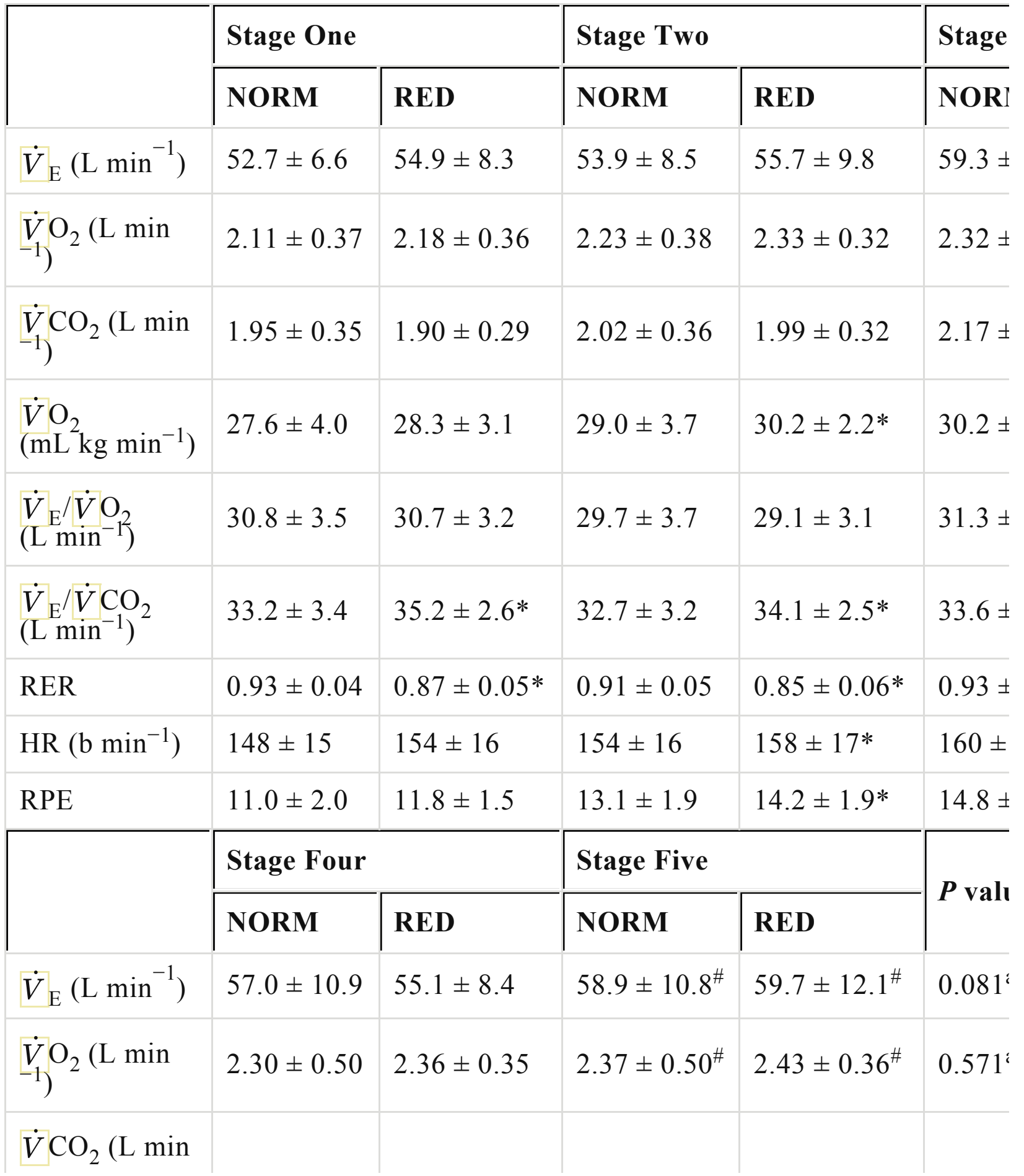




\begin{tabular}{|c|c|c|c|c|c|}
\hline-1$)$ & $2.08 \pm 0.45$ & $1.98 \pm 0.32$ & $2.12 \pm 0.45^{\#}$ & $2.06 \pm 0.38^{\#}$ & $0.439^{i}$ \\
\hline $\begin{array}{l}\dot{V} \mathrm{O}_{2}(\mathrm{~mL} \\
\left.\mathrm{kg} \mathrm{min}^{-1}\right)\end{array}$ & $29.9 \pm 4.6$ & $30.5 \pm 2.4$ & $30.7 \pm 4.4^{\#}$ & $31.4 \pm 2.6$ & $0.568^{i}$ \\
\hline $\begin{array}{l}\dot{V}_{\mathrm{E}} / \dot{V} \mathrm{O}_{2} \\
\left(\mathrm{~L}^{2} \min ^{-1}\right)\end{array}$ & $30.5 \pm 3.8$ & $28.3 \pm 2.1^{*}$ & $30.6 \pm 4.0$ & $29.6 \pm 3.0$ & $0.027^{1}$ \\
\hline $\begin{array}{l}\dot{V}_{\mathrm{E}} / \dot{\vec{V}} \mathrm{CO}_{2} \\
\left(\mathrm{~L} \mathrm{~min}^{-1}\right)\end{array}$ & $33.6 \pm 3.2$ & $34.0 \pm 2.0$ & $34.2 \pm 3.9$ & $35.2 \pm 2.1^{*}$ & $0.013^{1}$ \\
\hline RER & $0.91 \pm 0.05$ & $0.83 \pm 0.05^{*}$ & $0.89 \pm 0.04^{\#}$ & $0.84 \pm 0.06^{*}$ & 0.000 \\
\hline $\operatorname{HR}\left(\mathrm{b} \min ^{-1}\right)$ & $165 \pm 17$ & $165 \pm 16$ & $166 \pm 17^{\#}$ & $167 \pm 16^{\#}$ & $0.136^{i}$ \\
\hline RPE & $15.5 \pm 1.9$ & $15.8 \pm 1.9$ & $16.1 \pm 2.1^{\#}$ & $16.8 \pm 1.8^{*, \#}$ & $0.021^{\circ}$ \\
\hline
\end{tabular}

Values are mean $\pm \mathrm{SD}$

$\dot{V}_{\text {E }}$ minute ventilation, $\dot{V} \mathrm{O}_{2}$ oxygen uptake, $\dot{V} \mathrm{CO}_{2}$ carbon dioxide production, $\dot{V}$ equivalent of oxygen uptake, $\dot{V}_{\mathrm{E}} / \dot{V} \mathrm{CO}_{2}$ ventilatory equivalent of carbon dioxide respiratory exchange ratio, $H R$ heart rate, $R P E$ rating of perceived exertion

*-Difference between conditions, $t$ test

${ }^{\#}$ Difference between stages One and Five, $t$ test, $P<0.05$

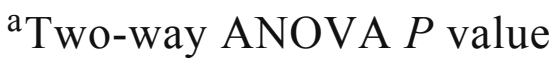

${ }^{\mathrm{b}}$ Friedman test $P$ value

\section{Respiratory exchange ratio}

RER during downhill running was significantly different between conditions $(P<0.05)$. For RED, the RER was lower from stage One $(0.87 \pm 0.05 ;$ NORM, $0.93 \pm 0.04 ; P<0.01, d=1.33$, large effect size) to stage Five $(0.84 \pm 0.06 ;$ NORM, $0.89 \pm 0.04 ; P<0.01, d=0.98$, large effect size), compared to NORM (Table 3 ). In NORM, RER was lower in stage Five compared to stage One (Table 3 ).

\section{Discussion}

Our main finding in this study was that performing a bout of aerobic, eccentric exercise with lowered glycogen, increased fat oxidation, bLa response, and effort perception, when compared to an eccentric bout with normal glycogen. Cardiorespiratory responses showed little difference 
between conditions; however, during the RED downhill run, either $\dot{V}_{\mathrm{E}}$ ' $\dot{V} \mathrm{CO}_{2}$ was elevated or $\dot{V} \mathrm{E} / \dot{V} \mathrm{O}_{2}$ was reduced. Increased bLa and altered ventilatory responses may reflect reliance upon type II fibres when performing muscle-damaging exercise with lowered muscle glycogen.

The present study used a cycling protocol that had previously been shown by Thomson et al. (1979) to cause $95 \%$ glycogen depletion in type I fibres in healthy men after $2 \mathrm{~h}$. Assuming similar type I glycogen depletion occurred in this study, our participants would be expected to commence the RED downhill run, recruiting predominantly type II fibres, which are more prone to eccentric-induced muscle damage (Fridén et al. 1983). This would agree with an earlier study (Krustrup et al. 2004), in which slowtwitch fibre depletion led to greater fast-twitch fibre recruitment and $\dot{V} \mathrm{O}_{2}$ for subsequent cycling exercise. We found similar $\dot{V}_{\mathrm{E}}, \dot{V} \mathrm{O}_{2}, \dot{V} \mathrm{CO}_{2}$ and HR responses between conditions, from stage One to Five. This may be attributed to the relatively low intensity of downhill running. For example, participants exercised between 62 and $65 \% \dot{V} \mathrm{O}_{2 \max }$ for glycogen reducing cycling, and between 51 and $59 \% \dot{V} \mathrm{O}_{2 \max }$ for downhill running. Therefore, the running intensity may not have been high enough to evoke distinguishable ventilatory responses between NORM and RED conditions. An eccentric protocol using higher intensities is likely to lead to greater muscle damage and delayed onset of muscle soreness (DOMS) (Paschalis et al. 2005). In this study we intended to investigate a whole-body eccentric-biased exercise common to both: day-to-day occupational activities, such as walking and stair descent, and endurance activities, such as marathon and cross-country running.

Previously, pre-exercise glycogen depletion has been shown to increase $\dot{V} \mathrm{O}_{2}$, without altering $\dot{V} \mathrm{O}_{2}$ kinetics for cycling (Bouckaert et al. 2004). After 9 min of high intensity cycling ( $85 \% \dot{V} \mathrm{O}_{2 \text { peak }}$ power), bLa increased to $8.1 \mathrm{mmol} \mathrm{L}^{-1}$ for normal glycogen, and $6.9 \mathrm{mmol} \mathrm{L}^{-1}$ for depleted glycogen. The end-of-exercise bLa values in this study were $1.98 \mathrm{mmol} \mathrm{L}^{-1}$ for NORM and $2.14 \mathrm{mmol} \mathrm{L}^{-1}$ for RED. These differences can be attributed to the exercise mode, intensity and duration. In particular, we found no change in $\dot{V} \mathrm{O}_{2}$ until stage Five of downhill running. Glycogen reduction protocols were similar between studies, but Bouckaert et al. (2004) used high intensity, short duration, concentric-biased exercise, whereas we used moderate intensity, prolonged, eccentric-biased exercise. In order to induce similar rates of $\dot{V} \mathrm{O}_{2}$ increase between 
contraction types, intensity must be high for eccentric exercise and low for concentric exercise (Perrey et al. 2001). As such, the $\dot{V} \mathrm{O}_{2}$ response to high intensity, concentric exercise, would be expected to be greater than that of moderate intensity, eccentric-biased downhill running. Using similar glycogen depleting exercise, others have reported increased $\dot{V}_{\mathrm{E}}$, and decreased $\dot{V} \mathrm{CO}_{2}$, for subsequent cycling exercise at moderate (55\% $\dot{V} \mathrm{O}_{2 \max }$ ) and heavy (95\% $\dot{V} \mathrm{O}_{2 \max }$ ) intensities (Segal and Brooks 1979). As expected, in a glycogen depleted state, heavy exercise was shown to elicit greater change in $\dot{V}_{\mathrm{E}}$ and $\dot{V} \mathrm{CO}_{2}$, than moderate exercise.

Interestingly, lactate response was unchanged for heavy exercise, but lower for moderate exercise. Unusual under normal circumstances, these findings were attributed to the remaining glycogen in type II fibres. For example, when exercising with depleted glycogen, the reduced glucose concentrations are typically accompanied by reduced lactate concentrations (Bouckaert et al. 2004; Hughes et al. 1982) due to a shift from glycolytic metabolism. Therefore, exercising with RED would cause lower circulating glucose and bLa. The current study showed reduced glucose, but increased bLa up to stage Four of downhill running with RED, compared to NORM. This may be due to greater muscle mass used for downhill running, and type II fibre recruitment, when compared to cycling exercise. Similar bLa from stage Four may be explained by RED involving higher type II recruitment, anaerobic glycolysis, and therefore bLa accumulation (stages One to Three), until further glycogen decrease, leading to a glycolytic to oxidative metabolic shift (stages Four to Five). Conversely, glycogen availability allowed sustained glycolysis for NORM, as glucose and lactate seemed to increase progressively throughout running. Elsewhere, Scott et al. (2003) found that eccentric contractions did not affect bLa during a subsequent bout of moderate intensity level running ( $\left.\sim 67 \% \dot{V} \mathrm{O}_{2 \max }\right)$. These authors concluded that predominant type II fibre damage would result in type I fibre recruitment for subsequent running. Type II fibre predominance (and therefore high glycogen utilisation) in our protocol was likely evident as NORM downhill running caused (1) a concomitant rise in $\dot{V} \mathrm{O}_{2}$ and lactate, and (2) an RER above 0.89 throughout. Our downhill running intensity was prescribed from individual lactate threshold, therefore if glycogen reduction cycling depleted mainly type I fibres (Thomson et al. 1979), our participants would be expected to commence downhill running using type II fibres. Downhill running also involves a large component of eccentric knee 
extensions, which would cause preferential type II fibre damage (Fridén et al. 1983 ).

Muscle soreness was not measured during downhill running, but effort perception increased from the first $(\sim 11.4$, light exertion) to final stages ( $\sim 16.5$, hard/very hard exertion) for both conditions. Effort perception was greater for RED for stages Two and Five, which, in part, agrees with higher RPE for $85 \% \dot{V} \mathrm{O}_{2 \text { peak }}$ cycling with glycogen depletion (Bouckaert et al. 2004). For submaximal running, DOMS is known to alter gait and increase energy cost (Braun and Dutto 2003). We found increased RPE during RED downhill running, suggesting greater perceived effort in a glycogen reduced state. This finding may reflect an earlier emergence of muscle damage during the RED downhill run. For example, Braun and Dutto (2003) suggested that the greatest damage occurs in the latter stages of downhill running due to altered gait kinematics. We are uncertain that glycogen reduction influenced running gait, and therefore muscle damage, in this study as biomechanical parameters were not examined. However, the potential effect of glycogen reduction on running biomechanics warrants further investigation, particularly for those working with endurance runners.

Performing heavy cycling exercise $\left(85 \% \dot{V} \mathrm{O}_{2 \max }\right)$ with glycogen depletion has been shown to increase $\dot{V} \mathrm{O}_{2}$, and decrease HR and RER (Krustrup et al. 2004). We showed lower RER, and similar $\dot{V} \mathrm{O}_{2}$ and HR for the RED downhill run, when compared to NORM. Decreased RER and decreased blood glucose concentration during the RED downhill run is indicative of greater fat oxidation. For a given concentric workload, increased fat metabolism in type I fibres elevates $\dot{V} \mathrm{O}_{2}$, to a greater degree than carbohydrate metabolism (Vøllestad et al. 1984; Yamanaka et al. 2012) Whether this is true for eccentric exercise remains to be seen, yet lower RER provides an indirect measure of our glycogen reduction protocol, as fat oxidation rate is determined by carbohydrate availability (Holloszy et al. 1998).

For low to moderate exercise intensities, $\dot{V}_{\mathrm{E}} / \dot{V} \mathrm{O}_{2}$ remains stable. As intensity increases, ventilation rises disproportionately and uncouples from $\dot{V} \mathrm{O}_{2}$, reflecting the ventilatory threshold. Beyond this threshold, the anaerobic metabolism is defined as an increase in $\dot{V}_{\mathrm{E}} / \dot{V}^{\mathrm{O}_{2}}$ and lactate, without change in $\dot{V}_{\mathrm{E}} / \dot{V} \mathrm{CO}_{2}$ (Caiozzo et al. 1982). At high intensities, 
increased $\dot{V}_{\mathrm{E}} / \dot{V} \mathrm{O}_{2}$ suggests ventilation cannot satisfy muscle oxygen delivery, and increased $\dot{V}_{\mathrm{E}} / \dot{V} \mathrm{CO}_{2}$ suggests ventilation cannot remove excess carbon dioxide. Higher $\dot{V}_{\mathrm{E}} / \dot{V} \mathrm{CO}_{2}$ commencing the RED downhill run was supported by increased bLa. In agreement, increased $\dot{V}_{\mathrm{E}} / \dot{V} \mathrm{CO}_{2}$ and decreased $\dot{V}_{\mathrm{E}} / \dot{V} \mathrm{O}_{2}$ have been shown for incremental cycling exercise with depleted glycogen (Hughes et al. 1982). Elsewhere, increased $\dot{V}_{\mathrm{E}} /$ $\dot{V} \mathrm{O}_{2}$ has been associated with greater effort perception when exercising with muscle damage (Twist and Eston 2009). Had RED resulted in greater hydrogen ion buffering during the downhill run, $\dot{V} \mathrm{CO}_{2}$, and, in turn, ventilation would increase. Although $\dot{V}_{\mathrm{E}}$ was similar between conditions, this would agree with higher initial bLa shown for RED downhill running. Ventilatory equivalent responses varied over running stages between conditions, partly due to fluctuations in $\dot{V}_{\mathrm{E}}, \dot{V} \mathrm{O}_{2}$ and $\dot{V} \mathrm{CO}_{2}$. For example, non-significantly higher $\dot{V}_{\mathrm{E}}$, and lower $\dot{V} \mathrm{CO}_{2}$, for RED (stages One, Two and Five) contributed to an increased $\dot{V}_{\mathrm{E}} / \dot{V} \mathrm{CO}_{2}$. Stabilised $\dot{V}_{\mathrm{E}}$, with a non-significantly higher $\dot{V} \mathrm{O}_{2}$, for RED (stages Three and Four) led to decreased $\dot{V}_{\mathrm{E}} / \dot{V} \mathrm{O}_{2}$.

Our protocol featured a recovery period between the glycogen reduction cycling and the downhill run of $\sim 10 \mathrm{~h}$. This interval allowed time for other confounding physiological disturbances, aside from glycogen reduction, associated with prolonged exercise (e.g., body temperature and hormonal alterations) to pass. Thus, for the downhill run we are confident that the effects of lowered muscle glycogen, and not the effects of other exerciseinduced perturbations, were being observed. The majority of glycogen repletion occurs within $3 \mathrm{~h}$ post-exercise (Maehlum and Hermansen 1978). Muscle glycogen content could not be directly measured, however, the following provide evidence that glycogen stores were lowered for the RED condition. First, blood glucose concentrations were substantially lower before and during the downhill run. Second, the RER was greatly reduced throughout the RED run, suggesting greater fat oxidation. Finally, a glycogen reduction protocol was used, shown to deplete glycogen in the knee extensor muscles of healthy men (Thomson et al. 1979).

Nevertheless, it needs to be acknowledged that the extent of glycogen depletion in the present study was based on a protocol with biopsyconfirmed glycogen levels. There is still the possibility that the extent of glycogen depletion in the present may differ from previous studies and it is a limitation that glycogen levels were not confirmed by direct biopsy 
observations.

This study demonstrated that downhill running performed with reduced muscle glycogen caused an attenuated blood glucose response, lowered RER, and in part, an increased blood lactate response. Cardiorespiratory responses were unchanged, yet ventilatory equivalents were somewhat altered, with glycogen reduction appearing to further stimulate carbon dioxide removal during downhill running. This is notable, given that relative exercise intensity was low and ventilatory equivalents were influenced by glycogen state, but not running duration. The alterations in $\dot{V}_{\mathrm{E}} / \dot{V} \mathrm{O}_{2}$ and bLa may suggest that carbon dioxide removal was somewhat impaired. Future work should determine whether lowered glycogen affects consistently the cardiorespiratory responses to more intense eccentric exercise. Evidence for initial type II recruitment during RED downhill running includes, (1) increased bLa (up to stage Three), and (2) increased $\dot{V}_{\mathrm{E}} / \dot{V} \mathrm{CO}_{2}$, and decreased $\dot{V}_{\mathrm{E}} / \dot{V} \mathrm{O}_{2}$. These findings have implications for recovery strategies of athletes performing new or heavy training, as well as demonstrating that glycogen reduction alters both ventilatory and metabolic responses to eccentric-biased exercise.

\section{Conflict of interest}

The authors declare no conflicts of interests.

\section{References}

Asp S, Daugaard JR, Richter EA (1995) Eccentric exercise decreases glucose transporter GLUT-4 protein in human skeletal muscle. J Physiol 482:705-712

Baumann CW, Green MS, Doyle JA, Rupp JC, Ingalls CP, Corona BT (2014) Muscle injury after low-intensity downhill running reduces running economy. J Strength Cond Res 28:1212-1218

Borg G (1998) Borg's perceived exertion and pain scales. Human Kinetics, Champaign

Bouckaert J, Jones AM, Koppo K (2004) Effect of glycogen depletion on the oxygen uptake slow component in humans. Int J Sports Med $25: 351-356$ 
Braun WA, Dutto DJ (2003) The effects of a single bout of downhill running and ensuing delayed onset of muscle soreness on running economy performed 48 h later. Eur J Appl Physiol 90:29-34

Caiozzo VJ, Davis JA, Ellis JF, Azus JL, Vandagriff R, Prietto CA, McMaster WC (1982) A comparison of gas exchange indices used to detect the anaerobic threshold. J Appl Physiol 53:1184-1189

Clarkson PM, Sayers SP (1999) Etiology of exercise-induced muscle damage. Can J Appl Physiol 24:234-248

Craig CL, Marshall AL, Sjöström M, Bauman AE, Booth ML, Ainsworth BE, Pratt M, Ekelund U, Yngve A, Sallis JF, Oja P (2003) International physical activity questionnaire: 12-country reliability and validity. Med Sci Sports Exerc 35:1381-1395

Eston RG, Mickleborough J, Baltzopoulos V (1995) Eccentric activation and muscle damage: biomechanical and physiological considerations during downhill running. Br J Sports Med 29:89-94

Eston RG, Lemmey AB, McHugh P, Byrne C, Walsh SE (2000) Effect of stride length on symptoms of exercise-induced muscle damage during a repeated bout of downhill running. Scand J Med Sci Sports 10:199-204

Faul F, Erdfelder E, Lang AG, Buchner A (2007) G*Power 3: a flexible statistical power analysis program for the social, behavioral, and biomedical sciences. Behav Res Methods 39:175-191

Fridén J, Sjostrom M, Ekblom B (1983) Myofibrillar damage following intense eccentric exercise in man. Int J Sports Med 4:170-176

Holloszy JO, Kohrt WM, Hansen PA (1998) The regulation of carbohydrate and fat metabolism during and after exercise. Front Biosci 15:1011-1027

Hughes EF, Turner SC (1982) Effects of glycogen depletion and pedaling speed on "anaerobic threshold". J Appl Physiol Respir Environ Exerc Physiol 52:1598-1607 
Krishnan RK, Evans WJ, Kirwan JP (2003) Impaired substrate oxidation in healthy elderly men after eccentric exercise. J Appl Physiol 94:716-723

Krustrup P, Soderlund K, Mohr M, Bangsbo J (2004) Slow-twitch fiber glycogen depletion elevates moderate-exercise fast-twitch fiber activity and O2 uptake. Med Sci Sports Exerc 36:973-982

Maehlum S, Hermansen L (1978) Muscle glycogen concentration during recovery after prolonged severe exercise in fasting subjects. Scand J Clin Lab Invest 38:557-560

Meyer K, Steiner R, Lastayo P, Lippuner K, Allemann Y, Eberli F, Schmid J, Saner H, Minetti AE, Moia C, Roi GS, Susta D, Ferretti G (2002) Energy cost of walking and running at extreme uphill and downhill slopes. J Appl Physiol 93:1039-1046

Minetti AE, Moia C, Roi GS, Susta D, Ferretti G. (2002) Energy cost of walking and running at extreme uphill and downhill slopes. J Appl Physiol 93:10391046 Newell J, Higgins D, Madden N, Cruickshank J, Einbeck J, McMillan K, McDonald R (2007) Software for calculating blood lactate endurance markers. J Sports Sci 25:1403-1409 move lower

O'Reilly KP, Warhol MJ, Fielding RA, Frontera WR, Meredith CN, Evans WJ (1987) Eccentric exercise-induced muscle damage impairs muscle glycogen repletion. J Appl Physiol 63:252-256

Osborne MA, Schneider DA (2006) Muscle glycogen reduction in man: relationship between surface EMG activity and oxygen uptake kinetics during heavy exercise. Exp Physiol 91:179-189

Paschalis V, Koutedakis Y, Jamurtas AZ, Mougios V, Baltzopoulos V (2005) Equal volumes of high and low intensity of eccentric exercise in relation to muscle damage and performance. J Strength Cond Res 19:184-188

Perrey S, Betik A, Candau R, Rouillon JD, Hughson RL (2001) Comparison of oxygen uptake kinetics during concentric and eccentric 
cycle exercise. J Appl Physiol 91:2135-2142

Scott KE, Rozenek R, Russo AC, Crussemeyer JA, Lacourse MG (2003) Effects of delayed onset muscle soreness on selected physiological responses to submaximal running. J Strength Cond Res $17: 652-658$

Segal SS, Brooks GA (1979) Effects of glycogen depletion and work load on postexercise O2 consumption and blood lactate. J Appl Physiol Respir Environ Exerc Physiol 47:514-521

Thomas JR, Salazar W, Landers DM (1991) What is missing in p less than.05? Effect size. Res Q Exerc Sport 62:344-348

Thomson JA, Green HJ, Houston ME (1979) Muscle glycogen depletion patterns in fast twitch fibre subgroups of man during submaximal and supramaximal exercise. Pflugers Archiv 379:105-108

Twist C, Eston RG (2009) The effect of exercise-induced muscle damage on perceived exertion and cycling endurance performance. Eur J Appl Physiol 105:559-567

Vøllestad NK, Vaage O, Hermansen L (1984) Muscle glycogen depletion patterns in type I and subgroups of type II fibres during prolonged severe exercise in man. Acta Physiol Scand 122:433-441

Widrick JJ, Costill DL, McConell GK, Anderson DE, Pearson DR, Zachwieja JJ (1992) Time course of glycogen accumulation after eccentric exercise. J Appl Physiol 72:1999-2004

Yamanaka R, Yunoki T, Arimitsu T, Lian CS, Roghayyeh A, Matsuura R, Yano T (2012) Relationship between effort sense and ventilatory response to intense exercise performed with reduced muscle glycogen. Eur J Appl Physiol 112:2149-2162 